\title{
Inhibition of the ATP-induced reactivation of demembranated hamster spermatozoa by the action of free $\mathrm{ATP}^{4-}$ and $\mathrm{MgATP}^{2-}$
}

\author{
C. H. Yeung \\ Max Planck Clinical Research Unit for Reproductive Medicine, Steinfurterstrasse 107, \\ D-4400 Muenster, Federal Republic of Germany
}

\begin{abstract}
Summary. Hamster spermatozoa collected from the caput and cauda epididymidis were washed, diluted in a medium containing Triton X-100 to dissolve the cell membrane and reactivated with various concentrations of $\mathrm{MgSO}_{4}$ and ATP. Stepwise increase in the concentrations of free $\mathrm{ATP}^{4-}$ from 0.08 to $1.1 \mathrm{mM}$ at constant concentrations of $\mathrm{MgATP}^{2-}$ caused a dose-dependent delay of reactivation but the maximal percentage of motile spermatozoa was inhibited only at $1.1 \mathrm{~mm}$. The inhibitory effect on caput spermatozoa was greater than that on cauda spermatozoa. When concentrations of ATP $^{4-}$ were fixed at $0.2 \mathrm{mM}, 2.9 \mathrm{mM}^{-\mathrm{MgATP}^{2-}}$ suppressed the reactivation of cauda spermatozoa. When compared to $0.9 \mathrm{mM}-\mathrm{MgATP}^{2-}$, reactivation of caput spermatozoa was delayed at $1.9 \mathrm{mM}$ and almost completely blocked by $2.9 \mathrm{mM}-\mathrm{MgATP}{ }^{2-}$. Inhibition of cauda sperm reactivation by $\mathrm{ATP}^{4-}$ and $\mathrm{MgATP}^{2-}$ were both prevented by the presence of trypsin $(50 \mathrm{ng} / \mathrm{ml})$. Incubation of cauda spermatozoa in the reactivation medium for 1 and 2 min before the addition of ATP progressively reduced the inhibitory effect of $\mathrm{ATP}^{4--}$; inhibition by $\mathrm{MgATP}^{2-}$ was reduced to a lesser extent. Addition of $100 \mu \mathrm{M}$-cyclic AMP to the medium abolished the delay of reactivation by $\mathrm{ATP}^{4-}$ but not that by $\mathrm{MgATP}^{2-}$. Before reactivation occurred, inhibitory concentrations of $\mathrm{ATP}^{4-}$ and $\mathrm{MgATP}^{2-}$ both induced large-angle coiling of sperm tails but in opposite direction to each other with reference to the asymmetric sperm head. The results suggest that free-ATP ${ }^{4-}$ and superoptimal concentrations of $\mathrm{MgATP}^{2-}$ inhibit flagellar movement by different mechanisms.
\end{abstract}

\section{Introduction}

Flagellar movement of spermatozoa is based on the active sliding of microtubules (see reviews by Satir, 1979; Brockaw, 1982) as a result of mechanochemical coupling of ATP hydrolysis by dynein ATPase (see review by Warner \& Mitchell, 1980) which is located periodically along the outer doublets of the $9+2$ axial filament structure of the sperm tail. Using the technique of extracting the membrane from spermatozoa with Triton X-100 and reactivating them with $\mathrm{Mg}^{2+}$ and ATP (Gibbons \& Gibbons, 1972), Yeung (1986) has demonstrated temporary inhibition of the initiation of reactivated motility of hamster spermatozoa by increasing the concentration of ATP from 1 to $3 \mathrm{~mm}$. This confirmed the observations of suboptimal reactivation of other mammalian spermatozoa when concentrations of ATP exceed the optimum (dog: Tash \& Means, 1982; ram: White \& Volgmayr, 1986) and may explain the discrepancies in the literature concerning the correlation of sperm motility with the intracellular concentrations of ATP (Rogers \& Morton, 1973; Foulkes \& MacDonald, 1979; Suter et al., 1979; Levin et al., 1981; Ke \& Tso, 1982; Calamera et al., 1982). In the present study the inhibitory action of ATP was further investigated. 


\section{Materials and Methods}

Sperm preparation. Mature male golden hamsters (Mesocricetus auratus, $90-130 \mathrm{~g}$ body weight, kept in $12 \mathrm{~h}$ light: $12 \mathrm{~h}$ dark with water and food-pellet intake ad libitum) were anaesthetized with urethane $(1.8 \mathrm{~g} / \mathrm{kg})$. Each epididymis was dissected in culture Medium 199 (Gibco, Paisley, U.K.); either the whole decapsulated caput region or a length (about $5 \mathrm{~cm}$ ) of unravelled distal cauda tubule was removed. Spermatozoa were released into Ca-free phosphatebuffered solution ( $\mathrm{pH} \mathrm{7.2)} \mathrm{from} \mathrm{coarsely} \mathrm{minced} \mathrm{caput} \mathrm{tubule} \mathrm{and} \mathrm{from} \mathrm{the} \mathrm{cauda} \mathrm{by} \mathrm{gently} \mathrm{squeezing} 1-\mathrm{cm}$ tubule segments. The spermatozoa were washed through $5 \%$ Ficoll $\left(M_{\mathrm{r}} 400000\right)$ in the phosphate buffer at $500 \mathrm{~g}_{\max }$ for $5 \mathrm{~min}$, resuspended in the buffer with sperm concentration adjusted to about $3 \times 10^{7} / \mathrm{ml}$ and kept on ice. Washed spermatozoa were used within $70 \mathrm{~min}$ of resuspension. Each suspension provided material for about 5 different treatments which were randomized with respect to their order of application in different experiments. All chemicals used were from Sigma Chemie (Munich, FRG) unless stated otherwise.

Demembranation and reactivation. A 3- $\mu$ sample of washed sperm suspension was diluted in $0.5 \mathrm{ml}$ of the demembranation and reactivation medium (Solution $\mathrm{R}$, containing $230 \mathrm{mM}$-sucrose, $15 \mathrm{~mm}$-potassium glutamate, $0.5 \mathrm{~mm}$ EDTA, $1 \mathrm{mg}$ polyvinylpyrolidone $\left(M_{\mathrm{r}} 40000\right) / \mathrm{ml}, 0.04 \%$ Triton X-100, $20 \mathrm{mM}-\mathrm{Tris}-\mathrm{HCl}$ adjusted to $\left.\mathrm{pH} 7.9\right)$ with various amounts of $\mathrm{MgSO}_{4}$. In some experiments, cyclic AMP $(100 \mu \mathrm{M})$, trypsin (type III-S; $50 \mathrm{ng} / \mathrm{ml}$ ) or soybean trypsin inhibitor (type I-S; $10 \mu \mathrm{g} / \mathrm{ml}$ ) was included in Solution R. Adenosine-5'-triphosphate (ATP; disodium salt, vanadium-free; Sigma Chemie or Boehringer Mannheim, Mannheim, FRG) was added after 1 or 2 min, or was already present in the medium when no preincubation time is indicated. The total concentrations of ATP and Mg needed to give the desired concentrations of free $\mathrm{ATP}^{4-}$ and $\mathrm{MgATP}^{2-}$ were calculated (Bartfai, 1979) using the association constants $\left(\log \mathrm{k}\right.$ ) of $4.04,6.32$ and 1.9 for $\mathrm{MgATP}^{2-}, \mathrm{MgEDTA}^{2-}$ and magnesium glutamate respectively (see reactivation conditions in Table 1).

Table 1. Total concentrations (mM) of ATP (disodium salt) $\left(\mathrm{ATP}_{1}\right)$ and $\mathrm{MgSO}_{4}\left(\mathrm{Mg}_{1}\right)$ added to Solution $\mathrm{R}$ to test the effects of increasing concentrations of free $\mathrm{ATP}^{4-}(\mathrm{A}-\mathrm{D})$ and $\mathrm{MgATP}^{2-}(\mathrm{E}-\mathrm{G})$ on the reactivation of demembranated hamster spermatozoa

\begin{tabular}{lccccc}
\hline $\begin{array}{l}\text { Reactivation } \\
\text { condition }\end{array}$ & ATP $^{4-}$ & MgATP $^{2-}$ & Mg $^{2+}$ & ATP $_{\mathbf{t}}$ & $\mathbf{M g}_{\mathbf{t}}$ \\
\hline A & 0.08 & 0.92 & 1.08 & 1.00 & 4.00 \\
B & 0.36 & 0.64 & 0.16 & 1.00 & 1.50 \\
C & 0.71 & 0.79 & 0.10 & 1.50 & 1.50 \\
D & 1.11 & 0.89 & 0.07 & 2.00 & 1.50 \\
E & 0.19 & 0.91 & 0.43 & 1.10 & 2.41 \\
F & 0.21 & 1.89 & 0.82 & 2.10 & 4.32 \\
G & 0.22 & 2.88 & 1.20 & 3.10 & 6.24 \\
$H^{*}$ & 0.08 & 1.02 & 1.15 & 1.10 & 4.31 \\
I* & 0.05 & 1.05 & 1.88 & 1.10 & 6.26 \\
\hline
\end{tabular}

*Controls for $\mathrm{Mg}^{2+}$ and $\mathrm{Mg}_{\mathrm{t}}$ as used in condition $\mathrm{G}$.

Measurements of motility. An aliquant of each reactivated sperm preparation was placed in an $80 \mu \mathrm{m}$ deep siliconized slide chamber (prepared as described by Yeung, 1984) and examined at room temperature $\left(21-25^{\circ} \mathrm{C}\right)$ using a Zeiss microscope with a $\times 10$ objective, condenser setting phase 3 and pseudo-dark-field optical conditions. Videorecording of each sample, in connection with a digital timer (time set at zero when spermatozoa were first exposed to exogenous ATP), was made by scanning across the chamber at random starting $1 \mathrm{~min}$ after the exposure of spermatozoa to ATP until the percentage of motile cells had apparently reached its peak and started to decline, or for $11 \mathrm{~min}$ when it was difficult to judge during this period of recording whether percentage motility had reached the maximum. Spermatozoa with oscillating flagella were counted as motile and percentage motility in each minute of recording was measured by counting all the cells (80-120) scanned. For each reactivation preparation, the time required to reach $75 \%$ of the maximal percentage motility was taken to reflect the overall rate of initiation of motility.

Statistical analysis. Statistical evaluation of the results was performed by analysis of variance and comparisons between treatment groups by partition of treatment sum-of-squares (Parker, 1979). Time intervals measured in integral minutes were transformed into their square-root values before analysis. Wilcoxon's Stratified test was used in the comparison of the sensitivity to the inhibitory effects of increasing concentrations of ATP between caput and cauda spermatozoa. 


\section{Results}

\section{Effect of free $A T P^{4-}$}

The majority of spermatozoa collected from the distal cauda and diluted into Medium 199 were motile (68.4 $\pm 4.5 \%$, mean \pm s.d., $n=5$ ). They were immediately immobilized in the demembranation solution but could resume regular flagellation in the presence of ATP depending on the reactivation conditions. In the first group of experiments, when the calculated concentrations of free $\mathrm{ATP}^{4-}$ present in the reactivation medium increased from 0.08 to $1.11 \mathrm{~mm}$, although those of $\mathrm{MgATP}^{2-}$ remained relatively constant at $0.64-0.92 \mathrm{~mm}$ (conditions A-D in Table 1 ), the rate of reactivation of the sperm preparation as a whole was decreased (Fig. 1). At the lowest concentration of $\mathrm{ATP}^{4-}$, over $75 \%$ maximal reactivation was always detected after 1 min exposure to ATP, but was delayed progressively with increasing concentrations of $\mathrm{ATP}^{4-}$ to $6.2 \pm 1.7 \mathrm{~min}$ (mean \pm s.d., $n=5$ ) at $1.11 \mathrm{mM}$. Maximal motility was slightly decreased at 0.71 and $1.11 \mathrm{~mm}$ $\operatorname{ATP}^{4-}(58.4 \pm 6.5$ and $61.4 \pm 7 \cdot 5 \%)$ when compared to values at 0.08 and $0.36 \mathrm{~mm}(77.0 \pm 4.5$ and $76.4 \pm 4.9 \%, P<0.05)$.

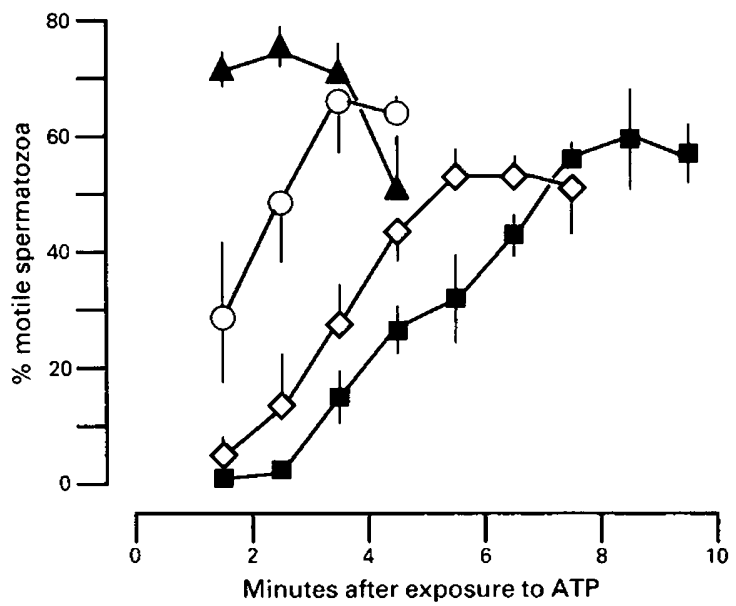

Fig. 1. Effect of free $\mathrm{ATP}^{4-}$ on the reactivation of demembranated spermatozoa from the hamster cauda epididymidis. Calculated concentrations of ATP4- were $0.08(\Delta), 0.36(O)$, $0 \cdot 71(\diamond)$ and $1 \cdot 11(\boldsymbol{\square}) \mathrm{mm}$ as in reactivation conditions A to D (Table 1) respectively. Values are mean \pm s.e.m. of 5 experiments.

In the second group of experiments, caput and cauda spermatozoa from each hamster were studied using the same conditions as in the first group. Delay in the reactivation of cauda spermatozoa was only detectable at ATP $^{4-}$ concentrations above $0.71 \mathrm{~mm}$ (Table 2, A-D) while concentrations of $\mathrm{ATP}^{4-}$ from 0.36 to $1.11 \mathrm{mM}$ exerted a greater inhibition on caput spermatozoa in the rate of attaining maximal reactivation (Wilcoxon's stratified test, $P<0.01$ ).

\section{Effect of $M g A T P^{2-}$}

When the concentration of $\mathrm{ATP}^{4-}$ was fixed at a low level of $0.2 \mathrm{mM}$, increasing $\mathrm{MgATP}^{2-}$ concentrations from 0.9 to $1.9 \mathrm{~mm}$ did not affect the reactivation of cauda spermatozoa, while a further increase to $2.9 \mathrm{~mm}\left(6.2 \mathrm{mM}-\mathrm{MgSO}_{4}\right)$ caused a marked decrease in maximal percentage reactivation and delayed the initiation of motility (E-G in Table 2). ATP from two different suppliers produced the same inhibitory effect. This was not observed when the total concentration of $\mathrm{MgSO}_{4}$ added remained the same $(6.2 \mathrm{mM})$ and $\mathrm{MgATP}^{2-}$ was reduced to $1 \mathrm{mM}$ (condition I, Tables $1 \& 2$ ). 
Table 2. Effects of increasing concentrations of $\mathrm{ATP}^{4-}$ and $\mathrm{MgATP}^{2-}$ on the reactivation of demembranated cauda and caput spermatozoa of hamsters (reactivation conditions as A to I in Table 1)

\begin{tabular}{|c|c|c|c|c|c|}
\hline \multirow{2}{*}{\multicolumn{2}{|c|}{$\begin{array}{l}\text { Reactivation } \\
\text { condition }\end{array}$}} & \multicolumn{2}{|c|}{ Maximal \% reactivation } & \multicolumn{2}{|c|}{ Reactivation time* $(\min )$} \\
\hline & & Cauda & Caput & Cauda & Caput \\
\hline \multicolumn{6}{|c|}{ mM ATP ${ }^{4-}$} \\
\hline A & 0.08 & - & $49 \cdot 0 \pm 6 \cdot 2^{\mathrm{a}}$ & - & $1 \cdot 2 \pm 0 \cdot 4^{a}$ \\
\hline B & $0 \cdot 36$ & $72 \cdot 9 \pm 5 \cdot 9^{a}$ & $60 \cdot 3 \pm 2 \cdot 0^{b}$ & $1 \cdot 2 \pm 0 \cdot 4^{a}$ & $2 \cdot 4 \pm 0 \cdot 5^{b}$ \\
\hline $\mathrm{C}$ & $0-71$ & $68 \cdot 3 \pm 9 \cdot 7^{a}$ & $47 \cdot 8 \pm 3 \cdot 2^{\mathrm{a}}$ & $3 \cdot 0 \pm 0 \cdot 6^{\mathrm{b}}$ & $4 \cdot 0 \pm 0 \cdot 6^{\mathrm{c}}$ \\
\hline D & $1 \cdot 11$ & $48 \cdot 9 \pm 9 \cdot 5^{b}$ & $28 \cdot 3 \pm 9 \cdot 5^{\mathrm{c}}$ & $3 \cdot 4 \pm 1 \cdot 0^{b}$ & $5 \cdot 2 \pm 0 \cdot 4^{d}$ \\
\hline \multicolumn{6}{|c|}{$\mathrm{mM} \mathrm{MgATP}^{2-}$} \\
\hline $\mathrm{E}$ & 0.91 & $84 \cdot 0 \pm 5 \cdot 6^{c}$ & $66 \cdot 1 \pm 8 \cdot 4^{\mathrm{d}}$ & $1 \cdot 4 \pm 0.6^{\mathrm{c}}$ & $2 \cdot 0 \pm$ \\
\hline $\mathrm{F}$ & 1.89 & $83 \cdot 1 \pm 8 \cdot 6^{\mathrm{c}}$ & $48 \cdot 3 \pm 6 \cdot 4^{\mathrm{e}}$ & $1 \cdot 6 \pm 0.6^{\mathrm{c}}$ & $3 \cdot 5 \pm 0.8^{f}$ \\
\hline G & $2 \cdot 88$ & $33 \cdot 0 \pm 19 \cdot 1^{d}$ & $4 \cdot 4 \pm 2 \cdot 4^{f}$ & $5 \cdot 7 \pm 2 \cdot 7^{\mathrm{d}}$ & $6 \cdot 3 \pm 1 \cdot 0^{f}$ \\
\hline $\mathrm{H}$ & 1.02 & - & $45 \cdot 5 \pm 6 \cdot 4^{c}$ & & $1 \cdot 6 \pm 0 \cdot 6^{c}$ \\
\hline I & 1.05 & $75 \cdot 5 \pm 7 \cdot 3^{\mathrm{c}}$ & - & $1 \cdot 2 \pm 0 \cdot 5^{\mathrm{c}}$ & - \\
\hline
\end{tabular}

Values are mean \pm s.d. of 5 experiments.

*Time required to achieve over $75 \%$ maximal reactivation after exposure to ATP; minimal time detectable was 1 min (see 'Methods').

Within columns and conditions $\mathrm{A}-\mathrm{D}$, and $\mathrm{E}-\mathrm{I}$, values with different superscript letters are significantly different $(P<0.05)$.

Table 3. Treatments affecting the inhibitory actions of $1.1 \mathrm{~mm}$-free ATP ${ }^{4}$ and $2.9 \mathrm{~mm}$ $\mathrm{MgATP}^{2-}$ on the reactivation of demembranated spermatozoa from the hamster cauda epididymidis

\begin{tabular}{|c|c|c|c|c|c|c|c|}
\hline \multirow{2}{*}{\multicolumn{2}{|c|}{ Treatment }} & \multicolumn{3}{|c|}{$1 \cdot 1 \mathrm{mM}-\mathrm{ATP}^{4-}$} & \multicolumn{3}{|c|}{$2.9 \mathrm{mM}-\mathrm{MgATP}{ }^{2-}$} \\
\hline & & $\%$ motility $\dagger$ & \multirow{2}{*}{$\frac{\operatorname{Min} \ddagger}{5 \cdot 5 \pm 1 \cdot 4^{a}}$} & \multirow[t]{2}{*}{$n$} & \multirow{2}{*}{$\frac{\% \text { motility } \dagger}{33 \cdot 0 \pm 19 \cdot 1^{a}}$} & \multirow{2}{*}{$\frac{\operatorname{Min}+}{6 \cdot 0 \pm 2 \cdot 8^{\mathrm{a}}}$} & \multirow{2}{*}{$\begin{array}{l}n \\
5\end{array}$} \\
\hline (a) & None* & $58 \cdot 0 \pm 9 \cdot 2^{\mathrm{a}}$ & & & & & \\
\hline (b) & 1 min preincubation & $50 \cdot 4 \pm 13 \cdot 4^{\mathrm{a}}$ & $2 \cdot 9 \pm 1 \cdot 1^{b}$ & 9 & $15 \cdot 7 \pm 10 \cdot 7^{\mathrm{a}}$ & $3.4 \pm 1.8^{\mathrm{b}}$ & 5 \\
\hline (c) & 2 min preincubation & $58 \cdot 1 \pm 7 \cdot 8^{\mathrm{a}}$ & $1 \cdot 3 \pm 0.5^{\mathrm{c}}$ & 7 & $24 \cdot 3 \pm 16 \cdot 2^{a}$ & $3 \cdot 2 \pm 1 \cdot 1^{b}$ & 5 \\
\hline (d) & + trypsin & $73 \cdot 3 \pm 4 \cdot 7^{b}$ & $1 \cdot 0 \pm 0.0^{\circ}$ & 4 & $79 \cdot 1 \pm 7 \cdot 6^{b}$ & $1.0 \pm 0.0^{c}$ & 5 \\
\hline (e) & + cAMP & $83.9 \pm 2.5^{b}$ & $2 \cdot 8 \pm 1 \cdot 1^{b}$ & 9 & $59 \cdot 2 \pm 10 \cdot 5^{b}$ & $5 \cdot 5 \pm 2 \cdot 4^{d}$ & 4 \\
\hline \multirow[t]{3}{*}{ (f) } & $\begin{array}{l}\text { I min preincubation } \\
\text { then }+ \text { cAMP }\end{array}$ & $82 \cdot 0+5 \cdot 2^{b}$ & {$\left[\cdot 3+0.7^{c}\right.$} & 7 & & & \\
\hline & $2 \min$ preincubation & & & & & & \\
\hline & in trypsin inhibitor & $38 \cdot 3 \pm 9 \cdot 7^{\mathrm{c}}$ & $2 \cdot 8 \pm 0 \cdot 7^{\mathrm{b}}$ & 6 & & & \\
\hline
\end{tabular}

Values are mean \pm s.d.

$\dagger$ Maximal \% motility during reactivation.

$\ddagger$ Time to achieve $>75 \%$ maximal reactivation after exposure to ATP.

${ }^{*}$ Control conditions for the study of factors affecting inhibitory actions of $\mathrm{ATP}^{4-}$ and $\mathrm{MgATP}^{2-}$ were as described in Table $\mathrm{I}, \mathrm{D}$ and $\mathrm{G}$, respectively.

Values within columns with different superscript letters are significantly different $(P<0.05)$.

On the other hand, an increase in $\mathrm{MgATP}^{2-}$ concentration from 0.9 to $1.9 \mathrm{~mm}$ did delay the reactivation of caput spermatozoa, which was almost completely suppressed at $2.9 \mathrm{~mm}$. When MgATP ${ }^{2}$ concentration was again maintained at $1 \mathrm{mM}$, free $\mathrm{Mg}^{2+}$ at a concentration equal to that in the above inhibitory condition had no effect on the initiation of motility although it slightly decreased the maximal percentage reactivation (Table 2, E \& H). A slight decrease in percentage motility of caput spermatozoa by high concentration of $\mathrm{Mg}^{2+}$ was also shown by reactivation conditions A and $\mathbf{B}$ (Table 2). 

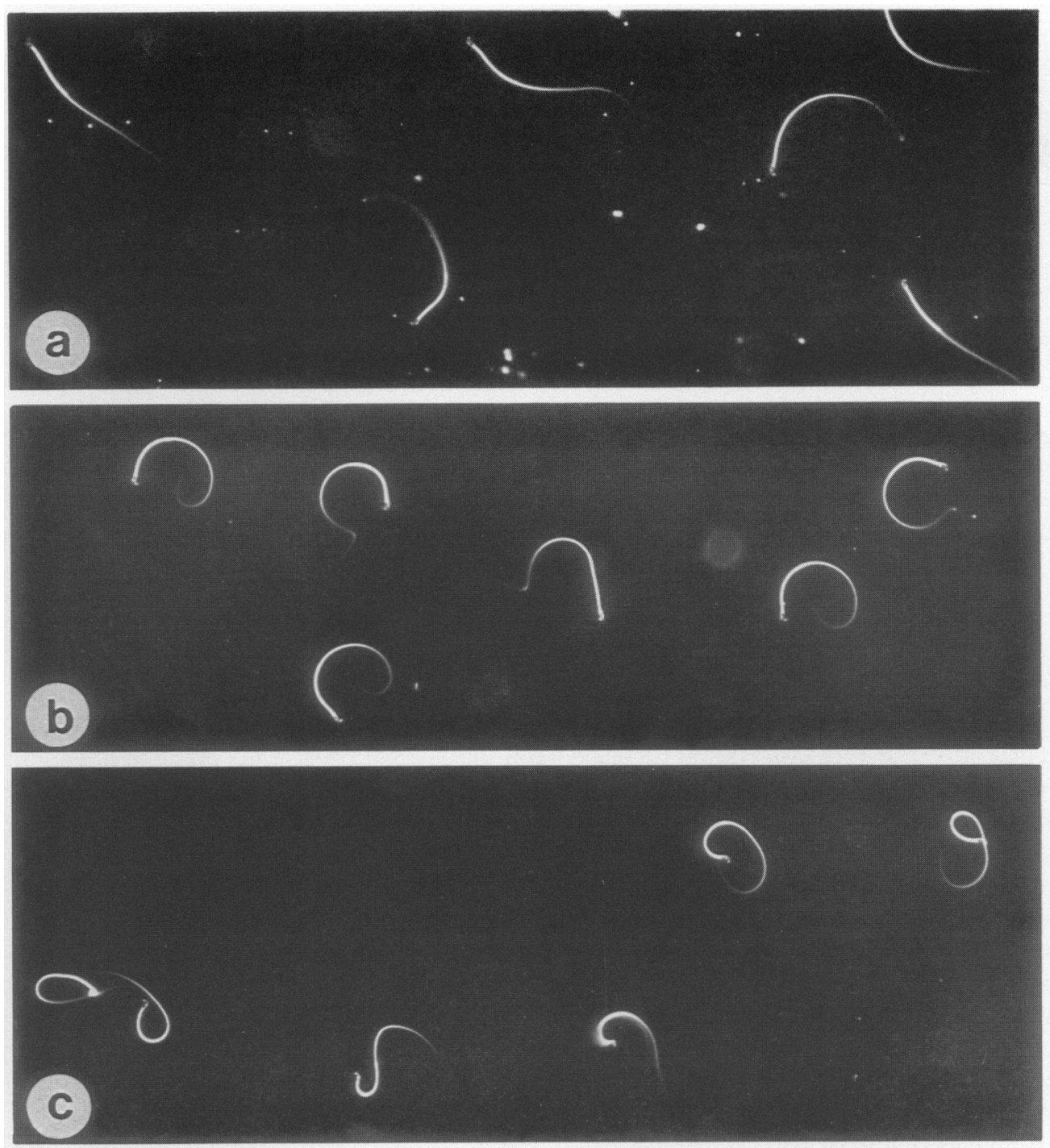

Fig. 2. Light micrographs of spermatozoa from the hamster cauda epididymidis in the demembranation-reactivation medium: (a) without the addition of ATP when the immotile sperm tails exhibited waves of slight curvatures; (b) in the presence of 1.1 mM-ATP ${ }^{4}$ (condition D in Table 1) when the whole or proximal two-thirds of the tail coiled in a large-angle $P$ bend; or (c) 2.9 mM-MgATP ${ }^{2-}$ (condition $G$ in Table 1) when coiling occurred in a direction opposite to that in (b), i.e. in $\mathrm{R}$ bends, before the initiation of regular motility, $\times 170$.

Factors affecting the inhibitory action of $A T P$ on the reactivation of cauda spermatozoa

A brief preincubation of cauda spermatozoa in Solution $\mathrm{R}$ for 1 and $2 \mathrm{~min}$ before the addition of ATP resulted in a stepwise recovery of the time course of reactivation in the presence of $1.1 \mathrm{~mm}$ $\mathrm{ATP}^{4-}$, and the inhibition of $2.9 \mathrm{mM}^{-\mathrm{MgATP}^{2-}}$ (i.e. $1 \mathrm{~mm}-\mathrm{MgATP}^{2-}$ in excess of its optimal concentration of $1.9 \mathrm{~mm}$ ) was reversed but to a lesser extent (Table 3 , treatments a $-\mathrm{c}$ ). The presence of trypsin inhibitor in the medium only partly blocked the effect of preincubation. Trypsin, however, allowed immediate maximal reactivation involving a higher percentage of motile spermatozoa 
Table 4. Number of cauda epididymal spermatozoa arrested in coils attaining or exceeding one revolution in the demembranation-reactivation medium containing $1.1 \mathrm{~mm}-\mathrm{ATP}^{4-}$ (A) or $2.9 \mathrm{mM}^{4} \mathrm{MgATP}^{2-}$ (B), expressed as percentage of immotile spermatozoa during a 2-min period before achieving maximal \% motility

\begin{tabular}{llccc}
\hline & & $\begin{array}{c}\text { No } \\
\text { treatment }\end{array}$ & $\begin{array}{c}1 \text { min } \\
\text { preincubation }\end{array}$ & + cAMP \\
\hline (A) & All R-bend coils $(n=9)$ & $19 \cdot 5 \pm 5 \cdot 1$ & $27 \cdot 7 \pm 10 \cdot 5$ & $81 \cdot 4 \pm 14 \cdot 4^{*}$ \\
(B) & All P-bend coils $(n=5)$ & $25 \cdot 1 \pm 8 \cdot 5$ & $47.9 \pm 8.0^{*}$ & $34.9 \pm 11 \cdot 0$ \\
\hline
\end{tabular}

Values are mean \pm s.d.

*Significantly different from no-treatment group $(P<0 \cdot 01)$.

than in its absence, although the duration of reactivated motility declined rapidly as described previously (Yeung, 1986).

Inhibition by $1.1 \mathrm{mM}^{-A T P^{4}-}$ was largely overcome in the presence of $100 \mu \mathrm{M}-\mathrm{cAMP}$, but inhibition by $2.9 \mathrm{mM}^{-\mathrm{MgATP}^{2}}{ }^{-}$in the initiation of reactivation persisted despite a partial recovery in the maximal percentage motility (Table 3 , treatment e).

\section{Flagellar waveforms under ATP inhibition}

Most cauda spermatozoa in the demembranation and reactivation solution in the absence of ATP were immobilized with a slightly curved flagellum (Fig. 2a) as previously described (Yeung \& Woolley, 1983). Before reactivation occurred, immotile spermatozoa in the presence of an inhibitory concentration of $\mathrm{ATP}^{4-}$ coiled into a large reverse (R) bend with a bend-angle reaching $360^{\circ}$ or more (the terminology of $P$ and $R$ bends is that defined by Woolley, 1977). This $R$ bend comprised the whole flagellum except when there was a short principal (P) bend at the neck and occasionally a small sharp distal $P$ bend which failed to propagate off the tip of the tail (Fig. 2b). This feature of 'sperm coils' was more marked when the medium contained cAMP (Table 4A). When these spermatozoa first became motile, the middle region of the tail straightened and beat irregularly. These intermittent bends initially did not propagate to the tip of the flagellum where the sharp $R$ bend curvature was retained. In about $0.5 \mathrm{~min}$ the whole flagellum was then recruited in regular and vigorous beating. Reactivated spermatozoa developed swimming patterns similar to those in optimal reactivation conditions with frequent display of a non-space-gaining motion referred to as 'looping' (Yeung, 1984).

Caput spermatozoa also exhibited $\mathrm{R}$ bends in response to increased concentrations of ATP4before motility was reinitiated. However, initial flagellation did not occur at mid-tail as in cauda spermatozoa, but only involved the distal half of the flagellum. The mid-piece remained stiff and did not flagellate with the principal piece under all reaction conditions.

In contrast to the effect of $\mathrm{ATP}^{4-}$, inhibition of reactivation by $\mathrm{MgATP}^{2-}$ induced the formation of 'sperm coils' in the opposite sense with respect to the hook of the head, resulting in a $P$ bend. In cauda and caput spermatozoa, the mid-piece gradually curved into this $\mathbf{P}$ bend, accompanied by slow propagation of the original $\mathrm{R}$ bend towards the tip of the flagellum, until the whole tail formed a coil often exceeding $360^{\circ}$ (Fig. 2c). This waveform persisted for a few minutes until the distal half of the tail began flagellation when reactivation started. Many coiled spermatozoa failed to develop beat cycles throughout the period of observation (up to $12 \mathrm{~min}$ ). Preincubation enhanced the curvature of such $P$ bend arrest (Table 4B). It was uncertain whether cAMP also enhanced the curvature because the arrest was brief, such that curvature might not have developed to the maximum before flagellation was initiated.

In the presence of trypsin, reactivation with the inhibitory concentration of either $\mathrm{ATP}^{4-}$ or $\mathrm{MgATP}^{2-}$ was immediate and no sperm coils appeared, except occasionally in preparations with 
$2.9 \mathrm{mM}^{-} \mathrm{MgATP}^{2-}$ in which motile spermatozoa retained a small sharp rigid $\mathbf{P}$ bend near the base of the flagellum.

\section{Discussion}

The substrate for dynein ATPase is $\mathrm{MgATP}^{2-}$. Alteration of the concentrations of total ATP in the reactivation medium should result in changes in the concentrations of $\mathrm{MgATP}^{2-}, \mathrm{ATP}^{4-}$ and $\mathrm{Mg}^{2+}$, all of which may be involved in the regulation of flagellar movement (Okuno \& Brokaw, 1979). Study of the individual effects of these chemical species on sperm motility is complicated by the dissociation equilibrium which makes it impossible to adjust the concentrations of one independently of the other two. In this study, the levels of total ATP throughout the incubation should be stable since hydrolysis by the low concentrations of spermatozoa, even when motile, is estimated from literature values to be less than $1 \%$. Suppression of reactivation by increasing the concentration of $\mathrm{MgATP}^{2-}$ at a constant and low concentration of $\mathrm{ATP}^{4-}$ was accompanied by an increase in $\mathrm{Mg}^{2+}$ concentrations. Although increasing concentrations of $\mathrm{Mg}^{2+}$ could decrease seaurchin sperm flagellation (Okuno \& Brokaw, 1979), neither the highest concentration of total $\mathrm{MgSO}_{4}$ nor that of $\mathrm{Mg}^{2+}$ tested was responsible for such effects when $\mathrm{MgATP}^{2-}$ was kept at low concentrations. Increase in the concentrations of $\mathrm{ATP}^{4-}$ in the presence of a relatively constant level of $\mathrm{MgATP}^{2-}$ also led to a progressive delay in the initiation of motility in demembranated caput and cauda spermatozoa. These findings agree with the previous results (Yeung, 1986) in which temporary inhibition of reactivation was obtained using $3 \mathrm{mM}-\mathrm{ATP}$ and $4 \mathrm{~mm}-\mathrm{MgSO}_{4}$, which should produce $0.51 \mathrm{mM}^{-\mathrm{ATP}^{4}-}$ and $2.5 \mathrm{mM}^{-\mathrm{MgATP}^{2-}}$.

Although dynein ATPase extracted from sea-urchin spermatozoa is inhibited by free ATP (Hayashi, 1976), neither ATP4- nor $\mathrm{MgATP}^{2-}$ in concentrations used in the present study on hamster spermatozoa appeared to exert their inhibitory action on motility directly on the active sliding of microtubules, since maximal reactivation occurred without delay in the presence of trypsin suggesting that the mechanisms involved are trypsin sensitive. However, these two inhibitory mechanisms are not the same, as indicated by the different characteristic flagellar waveforms induced and the ability of cAMP to overcome the blockade, particularly in the initiation of reactivation, by $\mathrm{ATP}^{4-}$ but not by $\mathrm{MgATP}^{2-}$.

Regulation of sperm motility by cyclic AMP is well known (see reviews by Garbers \& Kopf, 1980; Tash \& Means, 1983). Cyclic AMP is required for immediate reactivation of demembranated mature rat spermatozoa (Fentie \& Lindemann, 1978; Treetipsatit \& Chulavatnatol, 1982) and can enhance the motility of demembranated immature spermatozoa from hamsters (Yeung, 1984; Ishijima \& Mohri, 1985). Activation of sperm flagellation involves the cAMP-dependent phosphorylation of a specific axonemal protein, axokinin (Tash et al., 1984, 1986). The action of ATP4described here could be via the reduction of endogenous adenylate cyclase activity and the resultant reduced production of cAMP, although it has been demonstrated by enzyme-kinetic analysis that free ATP $^{4-}$ is not a strong inhibitor of bovine sperm adenylate cyclase if at all (Brown \& Casillas, 1986). Another possible indirect reduction in this enzyme activity by increasing ATP ${ }^{4}$ concentrations could result from the concommitant decrease in the concentrations of $\mathrm{Mg}^{2+}$, a stimulator for the enzyme. However, the delay in the initiation of reactivation was not as completely abolished by cAMP alone as by cAMP added after 1 min preincubation of the sperm preparation, suggesting that some other factors are involved, which may include the endogenous specific protease activity that regulates sperm motility (de Lamirande \& Gagnon, 1986). This unknown factor was not specifically sensitive to soybean trypsin inhibitor because the presence of the latter did not completely suppress the enhancing effect of preincubation.

The mechanism of the inhibitory effect of $\mathrm{MgATP}^{2-}$ is unclear. Failure of cAMP to completely overcome this inhibition suggests that the blockade of the initiation of bend cycles occurs before the step regulated by cAMP. 
The present observations for hamster spermatozoa are probably the manifestation of some general features of flagella and cilia. Superoptimal concentrations of ATP can arrest sliding of microtubules in Tetrahymena cilia (Warner \& Zanetti, 1980) and induce quiescence in sea-urchin spermatozoa (Brokaw, 1984). These phenomena, as well as the inhibitory effects of other factors on flagellar movement including calcium and lithium ions (Gibbons \& Gibbons, 1980, 1984), are also abolished by gentle trypsin disgestion. Further study of the induction of formation of 'sperm coils' in opposite senses of $\mathrm{ATP}^{4-}$ and $\mathrm{MgATP}^{2-}$ and the subsequent initiation of beat cycles may contribute to the understanding of bend initiation and the 'switch-point' mechanism of active sliding of microtubule postulated as the basis for bending cycles in cilia and flagella (see review by Satir, 1984).

While further investigation is required to elucidate the inhibitory mechanisms of these two ATP species, the present findings emphasize the subtlety of the regulation of sperm motility through ATP concentration as well as its various ionic forms.

I thank Professor E. Nieschlag and Dr T. G. Cooper for helpful comments on the manuscript.

\section{References}

Bartfai, T. (1979) Preparation of metal-chelate complexes and the design of steady-state kinetic experiments involving metal nucleotide complexes. $A d v$. cyclic Nucleotide Res. 10, 219-242.

Brokaw, C.J. (1982) Generation of the bending cycle in cilia and flagella. Cell Motility, Suppl. 1, 137-142.

Brokaw, C.J. (1984) Cyclic AMP-dependent activation of sea urchin and tunicate sperm motility. Ann. N.Y. Acad. Sci. 438, 132-141.

Brown, M.A. \& Casillas, E.R. (1986) Mn and Mn-ATP interactions with bovine sperm adenylate cyclase. Archs Biochem. Biophys. 224, 219-226.

Calamera, J.C., Brugo, S. \& Vilar, O. (1982) Relation. between motility and ATP in human spermatozoa. Andrologia 14, 239-241.

de Lamirande, E. \& Gagnon, C. (1986) Effects of protease inhibitors and substrates on motility of mammalian spermatozoa. J. Cell Biol. 102, 1378-1383.

Fentie, 1.H. \& Lindemann, C.B. (1978) Simultaneous comparison of motility and ATPase activity in Triton X-100 extracted rat sperm. J. Cell Biol. 79, 290a.

Foulkes, J.A. \& MacDonald, B.J. (1979) The relationship between ATP content and motility of bovine spermatozoa. Theriogenology 11, 313-319.

Garbers, D.L. \& Kopf, G.S. (1980) The regulation of spermatozoa by calcium and cyclic nucleotides. $A d v$. cyclic Nucleotide Res. 13, 251-306.

Gibbons, B.H. \& Gibbons, I.R. (1972) Flagellar movement and adenosine triphosphatase activity in sea urchin sperm extracted with Triton X-100. J. Cell Biol. 54, 75-97.

Gibbons, B.H. \& Gibbons, I.R. (1980) Calcium-induced quiescence in reactivated sea urchin sperm. J. Cell Biol. 84, 13-27.

Gibbons, B.H. \& Gibbons, I.R. (1984) Lithium reversibly inhibits microtubule-based motility in sperm flagella. Nature, Lond. 309, 560-562.

Hayashi, M. (1976) Inhibition of axoneme and dynein ATPase from sea urchin sperm by free ATP. Biochim. Biophs. Acta 422, 225-230.
Ishijima, S. \& Mohri, H. (1985) A quantitative description of flagellar movement in golden-hamster spermatozoa. J. exp. Biol. 114, 463-475.

Ke, Y.B. \& Tso, W.W. (1982) Variations of gossypol susceptibility in rat spermatozoa during spermatogenesis. Int. J. Fertil. 27, 42-46.

Levin, R.M., Shofer, J. \& Wein, A.J. (1981) ATP concentration of human spermatozoa: lack of correlation with sperm motility. Andrologia 13, 468-472.

Okuno, M. \& Brokaw, C.J. (1979) Inhibition of movement of triton-demembranated sea urchin sperm flagella by $\mathrm{Mg} 2+$, ATP, ADP and Pi. J. Cell Sci. 38, 105-123.

Parker, R.E. (1979) Introductory Statistics for Biology, 2nd edn. Edward Arnold, London.

Rogers, B.J. \& Morton, B. (1973) ATP levels in hamster spermatozoa during capacitation in vitro. Biol. Reprod. 9, 361-369.

Satir, P. (1979) Basis of flagellar motility in spermatozoa: current status. In The Spermatozoon, pp. 81-90. Eds D. W. Fawcett \& J. M. Bedford. UrbanSchwarzenberg Inc, Baltimore.

Satir, P. (1984) The generation of ciliary motion. $J$. Protozool. 31, 8-12.

Suter, D., Chow, P.Y.W. \& Martin, I.C.A. (1979) Maintenance of motility in human spermatozoa by energy derived through oxidative phosphorylation and addition of albumin. Biol. Reprod. 20, 505-510.

Tash, J.S. \& Means, A.R. (1982) Regulation of protein phosphorylation and motility of sperm by cyclic adenosine monophosphate and calcium. Biol. Reprod. 26, 745-763.

Tash, J.S. \& Means, A.R. (1983) Cyclic adenosine 3',5' mono-phosphate, calcium and protein phosphorylation in flagellar motility. Biol. Reprod. 28, 75-104.

Tash, J.S., Kaker, S.S. \& Means, A.R. (1984) Flagellar motility requires the cAMP-dependent phosphorylation of a heat-stable NP-40-soluble $56 \mathrm{kd}$ protein, axokinin. Cell 38, 551-559.

Tash, J.S., Hidaka, H. \& Means, A.R. (1986) Axokinin 
phosphorylation by cAMP-dependent protein kinase is sufficient for activation of sperm flagellar motility. J. Cell Biol. 103, 649-655.

Treetipsatit, N. \& Chulavatnatol, M. (1982) Effects of ATP, cAMP and $\mathrm{pH}$ on the initiation of flagellar movement in demembranated models of rat spermatozoa. Expl Cell Res. 142, 495-499.

Warner, F.D. \& Mitchell, D.R. (1980) Dynein: The mechanochemical coupling adenosine triphosphatase of microtubule-based sliding filament mechanisms. Int. Rev. Cytol. 66, 1-43.

Warner, F.D. \& Zanetti, C. (1980) Properties of microtubule sliding disintegration in isolated Tetrahymena cilia. J. Cell Biol. 86, 436-445.

White, I.G. \& Volgmayr, J.K. (1986) ATP-induced reactivation of ram testicular, cauda epididymal, and ejaculated spermatozoa extracted from Triton X-100. Biol. Reprod. 34, 183-193.
Woolley, D.M. (1977) Evidence for twisted plane undulation in golden hamster sperm tails. $J$. Cell Biol. 75, 851-865.

Yeung, C.H. (1984) Effects of cyclic AMP on the motility of mature and immature hamster epididymal spermatozoa studied by reactivation of the demembranated cells. Gamete Res. 9, 99-111.

Yeung, C.H. (1986) Temporary inhibition of the initiation of motility of demembranated hamster sperm by high concentrations of ATP. Int. J. Androl. 9, 359-370.

Yeung, C.H. \& Woolley, D.M. (1983) A study of bend formation in locally reactivated hamster sperm flagella. J. Muscle Res. Cell Motility 4, 625-645.

Received 30 January 1987 